\title{
Als Assistenzärztin bei der Versicherungsmedizin der Suva
}

\begin{abstract}
Gerlinde Heila, Philipp Habegger ${ }^{\text {, }}$, Regina Kunz ${ }^{\mathrm{c}}$
a Dr. med., war vom 1.3.2019 bis zum 29.2.2020 Weiterbildungsassistentin bei der Versicherungsmedizin der Suva; b Dr. med., war vom 1.1.2018 bis zum 31.12.2018 Weiterbildungsassistent bei der Versicherungsmedizin der Suva; ${ }^{c}$ Prof. Dr., Leiterin des Kompetenzzentrums Versicherungsmedizin der Suva in Luzern; Dozentin für Versicherungsmedizin, Universität Basel; Leiterin der Forschungsgruppe Evidence-based Insurance Medicine am Departement Klinische Forschung von Universität und Universitätsspital Basel
\end{abstract}

Gerlinde Heil und Philipp Habegger haben den Artikel gemeinsam konzipiert, der Beitrag gibt also die Erfahrungen beider Assistenzärzte wieder. Die Ich-Perspektive erklärt sich dadurch, dass Gerlinde Heil die Initiative für diesen Artikel ergriffen hatte und zudem die überwiegende Verantwortung beim Verfassen trug.
Die Versicherungsmedizin als medizinische Querschnittsdisziplin ist nur wenigen bekannt, trotz ihrer Bedeutung für Menschen mit gesundheitlichen Einschränkungen, für behandelnde Ärzte und die Gesellschaft. Im vorliegenden Artikel reflektiert eine Assistenzärztin über ihre Erfahrungen während ihres Weiterbildungsjahrs bei der Suva, der grössten Unfallversicherung der Schweiz.

Nach dem Medizinstudium und vier Jahren chirurgischer Weiterbildung wollte ich* Einblicke in die Versicherungsmedizin erhalten, weil mir als Ärztin in der Krankenversorgung ein gutes Verständnis unseres Sozialversicherungssystems wichtig war. Zwar fordern Weiterbildungsprogramme - z.B. in der Orthopädie oder der Psychiatrie - die Erstellung von Gutachten oder zumindest eine theoretische Ausbildung, allerdings geschieht dies mehrheitlich ausserhalb der Arbeitszeit und die Supervision kommt oftmals zu kurz. So wollte ich bei der Suva von erfahrenen Kollegen die Methodik der Begutachtung systematisch erlernen.

\section{Mit den Kreisärzten in der Agentur}

Im ersten Halbjahr arbeitete ich mit Kreisärzten in einer der 18 Suva-Agenturen. Zu den typischen Aufgaben der Ärzte gehören bei der Unfallversicherung u.a. Beurteilungen, ob gesundheitliche Einschränkungen auf ein versichertes Ereignis (d.h. einen Unfall) zurückzuführen sind (Kausalität), ob eine Arbeitsunfähigkeit vorliegt oder ob Leistungen wie Medikamente oder Hilfsmittel zweckmässig sind (Tab. 1). Mit ihren behandelnden Kollegen diskutieren Kreisärzte im Einzelfall auch spezifische Fragen.

Tabelle 1: Ärztliche Kernaufgaben im Kontext der Versicherungsmedizin [1].

Kernaufgaben

Beantworten von Fragen

\begin{tabular}{|c|c|}
\hline $\begin{array}{l}\text { 1. Beurteilung von Beeinträchtigungen und Verifizierung } \\
\text { deren Kausalität }\end{array}$ & $\begin{array}{l}\text {... über das Vorhandensein von gesundheitlichen Beeinträchti- } \\
\text { gungen und über deren Ursache (z.B. Unfall oder Krankheit). }\end{array}$ \\
\hline 2. Bestätigung der Arbeitsunfähigkeit («Krankschreibung») & $\begin{array}{l}\text {... ob und inwieweit Arbeitnehmer aufgrund von gesundheitlichen } \\
\text { Beeinträchtigungen nicht in der Lage sind, ihre beruflichen } \\
\text { Tätigkeiten zu verrichten. }\end{array}$ \\
\hline 3. Begutachtung von Langzeitarbeitsunfähigkeit & $\begin{array}{l}\text {... inwieweit Arbeitnehmer in der Lage sind, eine Form von geeig- } \\
\text { neter Arbeit zu verrichten, trotz vorhandener gesundheitlicher } \\
\text { Einschränkungen. }\end{array}$ \\
\hline 5. Massnahmen zur Förderung der Teilhabe & $\begin{array}{l}\text {... zur Wirksamkeit spezifischer Massnahmen zur Förderung der } \\
\text { Rückkehr an den Arbeitsplatz oder anderer gesellschaftlicher } \\
\text { Beteiligungen. }\end{array}$ \\
\hline 7. Risikobewertung für Privatversicherer & $\begin{array}{l}\text {... zum Risiko, dass eine Person aufgrund gesundheitlicher Ein- } \\
\text { schränkungen Versicherungsleistungen geltend macht. }\end{array}$ \\
\hline $\begin{array}{l}\text { 8. Zusammenarbeit mit behandelnden Kolleginnen } \\
\text { und Kollegen }\end{array}$ & $\begin{array}{l}\text {... zu spezifischen Fragen im Einzelfall. } \\
\ldots \text { zu übergeordneten versicherungsmedizinischen Themen. }\end{array}$ \\
\hline
\end{tabular}
zu übergeordneten versicherungsmedizinischen Themen. 
Kreisärzte sind beratend tätig. Mit ihrer umfangreichen klinischen Erfahrung in der Unfallmedizin beantworten sie medizinische Fragen, denen Fallführer spezialisierte Sachbearbeiter und Juristen - bei der Bearbeitung von Versicherungsfällen begegnen, und tragen somit zu einer korrekten Beurteilung des medizinischen Sachverhalts bei. Dabei beurteilen die Ärzte den medizinischen Verlauf und die Fortschritte in der Genesung, empfehlen weitere Massnahmen, die den Heilungsprozess fördern, und bestimmen im Verlauf den Zeitpunkt, ab dem die verunfallte Person durch weitere Massnahmen keine Besserung der Funktionalität mehr erwarten kann. Sie schätzen die gesundheitlichen Einbussen der versicherten Person und legen den Integritätsschaden fest, für den die Suva per Gesetz eine Geldleistung auszahlt. Der Gesetzgeber verpflichtet die Kreisärzte zur Unparteilichkeit, d.h., ihre Beurteilungen unterliegen keinen Vorgaben der Versicherung.

Meine vierjährige Erfahrung als chirurgische Assistenzärztin verhalf mir zu einem guten Einstieg. Ich kannte die gängigen Traumen, ihre Komplikationen und Behandlungsformen und war mit Medikamenten und Hilfsmitteln vertraut. Unter Supervision übertrugen mir die neuen Kollegen rasch die Verantwortung für eigene Fälle. Die Dokumente im Versichertendossier - Echtzeitdokumente zum Unfallgeschehen, Operationsberichte, Entlassungsbriefe und Bildgebung nachzuvollziehen und einzuordnen erfordert grosse klinische Expertise. Hier lernte ich sehr viel von den Kreisärzten. Ich fand es spannend, auch lange Behandlungsverläufe mittels medizinischer Dokumente nachzuvollziehen und mir Gedanken zu den Fragen der Fallführer zu machen (Tab. 2). Manche liessen sich zügig beantworten, z.B. ob ein Medikament unfallbedingt verschrieben wurde, andere erforderten eine vertiefte Beschäftigung mit dem Einzelfall, unfallmedizinische Erfahrung und versicherungsmedizinische Kenntnisse. Meist geht es um die Kausalität, also ob der Unfall «mit überwiegender Wahrscheinlichkeit» zu dem geltend gemachten Gesundheits-«Schaden» also Frakturen, Rupturen, Kopfschmerzen oder kognitiven Einschränkungen - geführt hat. Solche Fragen stellen sich bei Schäden an der Rotatorenmanschette oder bei Kopfverletzungen. In der Patientenversorgung, wo das diagnostische und therapeutische Vorgehen im Vordergrund steht, spielt die Frage der Kausalität nur eine untergeordnete Rolle. Mit der Frage nach der Ursache beklagter Beschwerden klärt die Unfallversicherung, ob die Unfall- oder die Krankenversicherung die Kosten übernehmen muss.

Bei unklaren Fallverläufen, Verzögerungen bei der Rückkehr zur Arbeit oder vor Fallabschluss laden die Fallführer verunfallte Personen zu einer kreisärztlichen Untersuchung in die Agentur ein, bei der sich die Ärzte ein eigenes Bild vom Heilungsverlauf machen. Hier habe ich gelernt, Versicherte zu ihren funktionellen Fähigkeiten bzw. Einschränkungen zu befragen und $\mathrm{zu}$ untersuchen. Typische Eingangsfragen adressierten z.B. die (maximal) mögliche Dauer eines Spaziergangs oder das eigenständige Tragen der Einkaufstasche.

Darüber hinaus war ich eingebunden in die "Rundgänge» bei den Sachbearbeitern, bei denen kürzere Fragen sofort im Gespräch geklärt wurden. Ich beteiligte mich an der interdisziplinären Schmerzsprechstunde, in der Fallführer, Kreisarzt, Neurologe und Psychiater gemeinsam die Situation des Versicherten aus unterschiedlichen Perspektiven beleuchteten. In einer Fortbildung zum Kniegelenk teilte ich mein medizinisches Wissen mit den Heilkostenspezialisten. Ein kleines Highlight war der Betriebsbesuch mit einem Aussendienstmitarbeiter, um die individuellen Arbeitsumstände einer verunfallten Person einzuschätzen und die Möglichkeiten der Wiedereingliederung zu beurteilen. Wir lernten den handwerklichen Arbeitsplatz des Versicherten kennen und überlegten gemeinsam mit dem Vorgesetzten, wie eine zukünftige Tätigkeit im Betrieb aussehen könnte.

\footnotetext{
Tabelle 2: Typische Fragen von Fallführerenden und Juristen an die beratenden Ärzte.

Kausalität Sind die geltend gemachten Beschwerden mit überwiegender Wahrscheinlichkeit auf den Unfall zurückzuführen?

War die Gesundheit der versicherten Person bei der vom aktuellen Unfallereignis betroffenen Körperregion mit überwiegender Wahrscheinlichkeit schon vor dem Unfall in stummer oder manifester Weise beeinträchtigt?

Fallverlauf Arbeitsfähigkeit:

Wie beurteilen Sie in Anbetracht der Unfallfolgen die Arbeitsfähigkeit als (... Beruf ...) in zeitlicher und leistungsmässiger Hinsicht?

Fallabschluss Behandlungsabschluss:

Ist die Behandlung (... N.N. ...) unfallbedingt noch notwendig?

Strukturelle Unfallfolgen:

Hat der Unfall mit überwiegender Wahrscheinlichkeit zu zusätzlichen strukturellen Läsionen geführt, welche objektivierbar sind?
} 


\section{Weiterbildungsjahr bei der Suva}

Die Versicherungsmedizin der Suva wurde vom Schweizerischen Institut für ärztliche Weiterund Fortbildung (SIWF) als Weiterbildungsstätte der Kategorie B zertifiziert: siwf-register.ch/ Detail.aspx?dossiernr=19954. Das SIWF erkennt ein Weiterbildungsjahr als Assistenzärztin oder -arzt in der Suva für den Facharzt für Prävention und Public Health an.

\section{Im Kompetenzzentrum Versicherungsmedizin}

Das zweite Halbjahr verbrachte ich am Kompetenzzentrum Versicherungsmedizin am Hauptsitz in Luzern. In den Fachgruppen Chirurgie/Orthopädie, Neurologie, Psychiatrie und Militärversicherung unterstützen Fachärzte mit diversen chirurgischen Subspezialitäten, Neurologen, Psychiater, Augenärzte u.a.m. die Fallführer und Juristen in medizinischen Fragen. Bei diesen Dossiers handelt es sich in der Regel um komplexere, oft interdisziplinäre Fälle oder Einsprachefälle, bei denen die verunfallte Person gegen die Verfügung der Suva Widerspruch erhoben hat. Einsprachen erfordern häufig ausführlichere Begründungen vonseiten der beratenden Ärzte auf die vorgebrachten Argumente. Zur Beantwortung der Fragen aus der Rechtsabteilung bei Einsprache oder Gerichtsfällen setzen sich die Ärzte - wie die Kreisärzte - erst mit dem Dossier auseinander, dann zusätzlich mit spezifischen Fachartikeln und versicherungsmedizinischer Literatur und erstellen eine ausführliche, oft mehrseitige Beurteilung mit wissenschaftlich fundierten Argumenten. Von den Kollegen erlernte ich bei umfangreicheren Beurteilungen ein systematisches Vorgehen, ihre Struktur, die relevanten Themen, die adressiert werden mussten, den Aufbau einer Argumentation in der Sprache medizinischer Laien und möglichst eindeutig formulierte Antworten. Anspruchsvoll waren die Beurteilungen bei Verunfallten mit schweren Verletzungen an mehreren Körpersystemen, die interdisziplinär bearbeitet wurden. So entstanden lehrreiche Diskussionen.
Ich hatte Gelegenheit zur Teilnahme an einer Visite im Schweizer Paraplegiker-Zentrum. Querschnittsverletzungen sind langwierig, aufwendig und häufig mit Komplikationen belastet. Bei diesen Visiten besprechen die Fallführer der Suva und die behandelnden Ärzte das aktuelle Management und die weitere Planung, unterstützt von spezialisierten Ärzten der Suva, die ihre Expertise bei Fragen zur Behandlung, zu benötigten Hilfsmitteln oder zur Kostengutsprache weiterer Massnahmen einbringen.

\section{Weitere Aktivitäten}

Neben meiner Tätigkeit unterstützte mich die Suva bei einem berufsbegleitenden CAS (Certificate of Advanced Studies) in Versicherungsmedizin am Swiss TPH/Universität Basel. In diesem multidisziplinären Studium lernte ich über die Organisation und Steuerung von Gesundheitssystemen, Gesundheitsökonomie, Sozial-/ Privatversicherung, Krankenversicherung und die Funktion des Vertrauensarztes, Public Health und Prävention. Ich vertiefte mein Verständnis über die vielen Facetten meiner Arbeit, erhielt juristische Grundkenntnisse zum Sozialversicherungswesen und zur weitreichenden Bedeutung eines gut funktionierenden Sozialversicherungssystems für die Risiken, denen wir in einer schnelllebigen Zeit ausgesetzt sind. Besonders wertvoll fand ich den Austausch mit Studienkollegen, die in der Risikoprüfung bei einer Versicherung, als Anwälte oder Vertrauensärzte tätig waren.

\section{Literatur}

1 de Boer W, Brage S, Kunz R. Versicherungsmedizin in klinisch-epidemiologischer Hinsicht: Ein Konzeptpapier. Suva Medical 2020 (09).

\section{Danksagung}

Wir danken den Teams der Agentur Zentralschweiz und des Kompetenzzentrums, den Ärzten, Assistierenden, den Sachbearbeitern und Juristen für die umfangreiche Begleitung und Unterstützung und die grosszügige Weitergabe ihres umfangreichen Wissensfundus. Wir dürfen auf ein Weiterbildungsjahr voller wertvoller Erfahrungen zurückblicken. Wir danken Christoph Bosshard und Robert Lang für kritisches Feedback zum Artikel.
Dr. Gerlinde Heil gerlindeheil[at]icloud.com

Dr. Philipp Habegger philipp.habegger[at]suva.ch

Prof. Dr. Regina Kunz regina.kunz[at]suva.ch

\section{Das Wichtigste in Kürze}

- Gerlinde Heil und Philipp Habegger haben sich während ihrer Facharztausbildung bei der Suva intensiv mit versicherungsmedizinischen Konzepten und juristischen Grundsätzen des Sozialversicherungssystems beschäftigt.

- Durch den Perspektivenwechsel hat sich ihr Blick auf das Schweizer Gesundheitswesen erweitert.

- Sie finden, dass sich das Jahr auch für ihre Tätigkeit in der Patientenversorgung gelohnt hat.

- Besonders positiv empfanden sie das kollegiale Miteinander, die flexiblen Arbeitszeiten und die abwechslungsreichen Arbeitsinhalte.

\section{L'essentiel en bref}

- Pendant leur formation de médecins spécialistes à la Suva, Gerlinde Heil und Philipp Habegger ont étudié de manière intensive les concepts de médecine des assurances et les principes juridiques du système d'assurances sociales.

- Ce changement de perspective a élargi leur vision du système de santé suisse.

- À leurs yeux, cette année leur a également été utile pour leur activité de prise en charge des patients.

- Ils ont particulièrement apprécié la coopération collégiale, les horaires flexibles et la diversité des contenus de travail. 